\title{
Real Time Safety Monitoring: Concept for Supporting Safe Flight Operations
}

\author{
Lilly Spirkovska* \\ NASA Ames Research Center, Moffett Field, CA 94035 \\ Indranil Roychoudhury ${ }^{\dagger}$ \\ Stinger Ghaffarian Technologies Inc., NASA Ames Research Center, Moffett Field, CA 94035 \\ Matthew Daigle ${ }^{\ddagger}$ and Kai Goebel ${ }^{\ddagger}$ \\ NASA Ames Research Center, Moffett Field, CA 94035, USA
}

\begin{abstract}
Processes, procedures, regulations, and technologies are continuously evolving to maintain or improve the safety of the National Airspace System (NAS). In this paper, we describe a Real Time Safety Monitoring (RTSM) system that benefits from these efforts to define a set of safety metrics that are automatically monitored in real-time. In addition to providing information about current potentially adverse conditions to a variety of users, from those who need a broad overview of a day's flight operations to those who need to decide on a control tactic to employ in the next five minutes, the RTSM system predicts conditions within a specified prediction horizon. Its intelligent interface alerts the user, presenting the information as appropriate considering the current context and circumstances. We illustrate the system concept with five conceptual use cases, describing which safety metrics may be of the most interest to five user groups and suggesting a multimodal display format. We posit that having access to information about adverse conditions in time to make efficient preemptive decisions without sacrificing safety will improve the already high level of safety and aid in the expansion planned for the NAS under the Next Generation Air Transportation System (NextGen).
\end{abstract}

\section{Introduction}

Flying in the National Airspace System (NAS) did not reach its current level of safety by accident. Numerous approaches have evolved over the past hundred years to improve the safety of flight operations, including safer aircraft and avionics designs, incorporation of human factors considerations, and improved training. A critical approach to improving safety is learning from accidents, incidents, and close-calls. Lessons learned are shared with the aviation community and, when appropriate, rules and regulations are enacted to prevent similar occurances in future operations. A number of organizations participate in the analysis effort, including the following:

- The National Transportation Safety Board (NTSB) ${ }^{1}$ maintains a database of and is the primary investigator of aviation accidents.

- NASA's Aviation Safety Reporting System team (ASRS) ${ }^{2}$ analyzes incident and close-call reports, identifying deficiencies and discrepancies before they lead to accidents.

- The FAA recently implemented the Aviation System Information Analysis and Sharing (ASIAS) ${ }^{3}$ system to facilitate analyses.

*NASA Ames Research Center, Moffett Field, CA 94035

${ }^{\dagger}$ SGT Inc, NASA Ames Research Center, Moffett Field, CA 94035, AIAA Member

$\ddagger$ NASA Ames Research Center, Moffett Field, CA 94035, AIAA Member 
- The FAA's Integrated Safety Analysis Modeling (ISAM) ${ }^{4}$ team uses accident and incident data to determine risk increases due to various hazards.

- Commercial airlines implement Safety Management Systems (SMS) ${ }^{5}$ to identify new hazards and evaluate the effectiveness of implemented risk management strategies.

- The Aircraft Owners and Pilots Association (AOPA) ${ }^{6}$ analyzes general aviation (GA) accidents and incidents and promotes techniques to improve GA safety.

Generally, these organizations analyze current NAS operations and, based on this data, recommend potential improvements to safety, measured by metrics ranging from deviation from standard procedures to fatal accident rates. It can take from a few months to several years for new recommendations to percolate through these processes.

Complementing these investigative analyses are processes supporting aeronautical decision making during operations. For example, the FAA's 3-P Risk Management process ${ }^{7}$ asserts that aeronautical decision making can be improved by following the basic steps: (i) Perceive hazards, (ii) Process to evaluate the level of risk, and (iii) Perform risk management. To perceive the gamut of potential hazards, defined as the "events, objects, or circumstances that could contribute to an undesired event", a pilot can apply the PAVE mnemonic and consider hazards within the categories of Pilot, Aircraft, enVironment, and External pressures. A similar concept of evaluating hazards as part of risk management is the FAA's Flight Risk Assessment Tool (FRAT),$^{8}$ adapted by the AOPA into the Flight Risk Evaluator online tool. ${ }^{9}$ Both tools lead a pilot through a checklist of potential hazards. Depending on the pilot's answers, the analysis aims to improve the pilot's situational awareness of any adverse conditions and culminates in a number that summarizes risk. If the total risk number exceeds the pilot's personal maximum acceptable risk, the pilot should consider appropriate mitigation strategies. These checklists can be used as part of a continuous risk management strategy (such as the 3-P Risk Management process), starting during preflight preparation and continuing in flight. However, it is a manual process and the pilot is responsible for pulling the necessary data at the appropriate time while also flying the plane, navigating, communicating, and managing aircraft systems.

An alternative approach to preventing accidents utilizes automated tools to augment the pilot and the air traffic controller. Example functionality provided by these tools include alerts for airspace incursion, traffic conflicts, terrain collision, runway overrun, and aircraft go-around detection. ${ }^{10}$ Typically, these tools narrowly target one hazard by evaluating incoming data and warning the user if very specific conditions are met. They have a short look-ahead and and serve to alert the user to react immediately to avoid an incident or accident. Essentially, they are safety nets for when standard procedures fail.

NAS operations have reached an unprecedented high level of safety ${ }^{11}$ through these processes, procedures, and tools that eliminate risks when possible or help pilots and controllers manage those risks that cannot be eliminated. Whether these approaches will suffice to protect future NAS operations is unclear. First, the number of NAS operations is forecast to increase, ${ }^{12}$ so if nothing is done to improve the accident rate, there is a possibility that the safety level will decrease below society's tolerance. ${ }^{a}$ Moreover, additional types of risk will be introduced with the increased number of operations by unmanned aircraft systems (UAS). ${ }^{14}$ Finally, concepts for the Next Generation Air Transportation System (NextGen) include reallocating responsibilities between air traffic controllers and airspace users; for example, transferring responsibility and authority from controllers to pilots and dispatchers for route selection and separation from other aircraft. ${ }^{15}$

To maintain or improve the current level of safety in light of these issues, we believe that there is a need for additional tools to support operations. In NAS operations, being just in the moment is not sufficient; pilots, controllers, and others providing real-time flight support must continuously look forward and anticipate the need for action. Awareness of the big picture and anticipation of how it may evolve informs decision making, and timely, well-informed decisions may lessen the impact on efficiency and on predictability of operations, two key performance indicators of the NAS. ${ }^{16}$ In the well-known Observe, Orient, Decide, Act (OODA) decision making loop, ${ }^{17}$ the Observe and Orient steps have been shown to be most critical, and failure to obtain needed information about adverse conditions and interpret it correctly, rather than the ability to decide on a course of action, is a major cause underlying aviation accidents. ${ }^{18-20}$ Toward that end, we propose a system, termed Real-Time Safety Monitoring (RTSM), that improves situational awareness by

\footnotetext{
${ }^{a}$ As explained in Skybrary: ${ }^{13}$ "Absolute safety is generally an unachievable and very expensive goal. Therefore the concept of acceptable safety has been adopted in risk bearing industries, including aviation. The term "acceptable risk" describes an event with a probability of occurrence and consequences acceptable to the society, i.e. the society is willing to take or be subjected to the risk that the event might bring."
} 
monitoring current and forecast conditions that can expose flights to harm and by depicting increased risk areas in a context-appropriate method. This information can enable collaborative, proactive management of aviation hazards. The system combines two technologies to automatically assess the airspace: (1) prognostics under uncertainty and (2) intelligent interfaces incorporating domain knowledge and user preferences. This assessment of the airspace enables more advanced planning and lookahead regarding existing or potential hazards, customized to support each user's perspective and goals. The proposed system applies the lessons learned of the previously mentioned risk management approaches to determine what to monitor. Further, in contrast to the conflict detection tools previously mentioned, it is not envisioned to be just a safety net, but rather a tool that will help NAS operators anticipate well in advance the evolution of operations and devise a plan to preemptively avoid hazards.

In this paper, we present a concept for the RTSM system to support safe flight operations. Here, we focus on describing the problem we are solving for the customer, enumerating the potential customers, and illustrating how RTSM could benefit selected customers. In a companion paper, ${ }^{21}$ we describe a prototype implementation of RTSM, including the software architecture and algorithms. In Section II, we define what we mean by "real-time" and "safety", categorize and describe safety metrics that promote safe flight operations, and provide a high-level description of the system architecture. In Section III, we enumerate the participants involved in flight operations and provide sample use cases on how the proposed system can support them. We employ these conceptual use cases as a means of communicating with stakeholders to elicit the detailed requirements for RTSM. We will seek expert opinions on such details as which hazards would be useful to predict and in what context, which hazards to present individually and which to combine, and under which conditions to alert of a hazard and the degree of alert they consider appropriate. Finally, in Section IV, we conclude with a summary and discussion of ongoing work.

\section{Real Time Safety Monitoring: Definitions, Metrics, and Architecture}

In this section, we define what we mean by "real time" and by "safety", discuss safety metrics, and provide a high-level architecture of RTSM. The dictionary definition of real time is "of or relating to applications in which the computer must respond as rapidly as required by the user or necessitated by the process being controlled [emphasis added]." 22 Within the context of flight operations, "real time" does not necessary imply instantaneous response. Rather, the required computer response time will vary for each user and each circumstance and is a function of the desired decision-making timeframe in that context. For example, the Air Traffic Control System Command Center (ATCSCC) planners do not reconsider a flow control strategy each time a flight departs from any airport. They instead need a summary of forecast airspace demand within some fairly loose tolerance (e.g., 15 min intervals), and decisions are updated perhaps once per hour. In contrast, a flight controller needs awareness of the traffic situation within a very tight tolerance to ensure safe but not excessive separation between flights, and decisions are updated with each surveillance update ( 1 sec to $12 \mathrm{sec}$, depending on the surveillance method). In the first case, 'real time' means 'within an hour'. In the latter case, 'real time' means at 'each surveillance update', with faster update rates enabling closer, and still safe, operations. Another term which could be substituted for real time is in time. Information needs to be provided to a particular user in time to support the user's task(s).

For RTSM, the tasks of interest are those which contribute to safe operations. Turning again to the dictionary, safe is defined as "protected from or not exposed to danger or risk; not likely to be harmed or lost." There is a famous saying by John A. Shedd that "A ship in harbor is safe, but that is not what ships are built for." (Arguably, ships are not even completely safe in harbor.) The same can be said of airplanes. Some level of risk must be accepted to fly. The level of acceptable flight risk - the likelihood and severity of harm - is determined by society and is a trade-off with reward. This leads to the International Civil Aviation Organization (ICAO) and FAA definition of safety as "the state in which the possibility of harm to persons or damage to property is reduced to, and maintained at or below, an acceptable level through a continuing process of hazard identification and risk management," ${ }^{23}$ with hazard defined as "the condition or circumstance that can lead to physical injury or damage."

How is safety measured? One measure of safety is the number of incidents ${ }^{\mathrm{b}}$. Incidents are often the result of a sequence of events that line up in an unfortunate way such that none of the existing mitigation strategies prevents the incident. If an incident does not occur, it does not imply that each of the events

\footnotetext{
${ }^{\mathrm{b}}$ The NTSB makes a distinction between accidents and incidents based on the degree of injury or property damage. Both imply a breach of safety. We use just the term incident to refer to either.
} 
in the sequence is safe. Rather, it is an illustration of the many checks and balances in aviation that have evolved over the decades to ensure safety under diverse, and possibly adverse, circumstances.

So few incidents occur in even a decade, it is challenging to generalize the situations into actionable lessons learned and encode them into additional checks and balances. However, incidents can be categorized, using the ICAO/CAST ${ }^{\mathrm{c}}$ taxonomy, ${ }^{24}$ for example, to aid incident prevention analyses. Such analyses can extract at least some of the contributing factors to incidents, that is, hazards to safe flight. Such contributing factors are the starting point for RTSM.

As discussed previously, lack of cognizance about hazards is a bigger contributor to incidents than faulty decision making. Thus, to further improve safety, RTSM monitors the NAS and alerts its users to any current or anticipated hazards. Subject matter experts, lessons learned from previous research, and accident, incident, and close calls reports were used to determine which hazards to monitor. The risk associated with a particular hazard is user and task dependent. For example, a strong tailwind is an asset to a flight enroute, but a liability on landing. Similarly, light icing is a liability to a small aircraft, but easily handled by a heavy aircraft with deicing capabilities. To account for these differences in what is considered harmful to a particular operation, we combine the hazard with domain knowledge and a threshold delineating what the particular user (or user group) considers the edge of an acceptable level of safety. The end result is a list of safety metrics representing the measures of the probability of encountering specified hazards.

Table 1: Safety Metrics

\begin{tabular}{|c|c|c|}
\hline Safety Metric & ICAO/CAST Taxonomy & Example Consolidated Safety Metrics \\
\hline $\begin{array}{l}\text { Prob. of Adverse } \\
\text { Weather Encounter }\end{array}$ & $\begin{array}{l}\text { ICE, FUEL (induction ice), } \\
\text { LOC-G (contaminated } \\
\text { runway), LOC-I, TURB, } \\
\text { UIMC, WSTRW }\end{array}$ & $\begin{array}{l}\text { Thunderstorms, turbulence, icing, wind, extreme } \\
\text { heat/cold, (low) visibility, (low) ceiling }\end{array}$ \\
\hline $\begin{array}{l}\text { Prob. of Airspace } \\
\text { Conflict }\end{array}$ & ATM & $\begin{array}{l}\text { SUA, TFR, pilot/controller training areas, noise } \\
\text { restriction areas }\end{array}$ \\
\hline $\begin{array}{l}\text { Prob. of Malfunctioning } \\
\text { Equipment Encounter }\end{array}$ & ATM & $\begin{array}{l}\text { Guidance (ILS, VASI/PAPI, lights), navigation } \\
\text { (radar, ADS-B, navaids), communication } \\
\text { (air-to-ground, ground-to-ground) }\end{array}$ \\
\hline $\begin{array}{l}\text { Prob. of Runway } \\
\text { Excursion }\end{array}$ & $\mathrm{RE}$ & $\begin{array}{l}\text { Contamination (snow, ice, wet), weather } \\
\text { (crosswind, tailwind), length, FOD }\end{array}$ \\
\hline $\begin{array}{l}\text { Prob. of Ground } \\
\text { Conflict }\end{array}$ & ADRM, GCOL & $\begin{array}{l}\text { Hotspots, controller observability, jetblast risk } \\
\text { areas, taxi complexity }\end{array}$ \\
\hline $\begin{array}{l}\text { Prob. of Airborne } \\
\text { Conflict }\end{array}$ & MAC & $\begin{array}{l}\text { Wake turbulence, congestion, traffic homogeneity, } \\
\text { probability of controller/pilot error }\end{array}$ \\
\hline $\begin{array}{l}\text { Prob. of Excessive Pilot } \\
\text { Workload, Prob. of } \\
\text { Excessive Controller } \\
\text { Workload }\end{array}$ & ATM, OTHER & $\begin{array}{l}\text { Traffic dynamic density, traffic homogeneity } \\
\text { (light/heavy/helicopter/glider/VFR/IFR, etc.), } \\
\text { equipment status, weather, degree of operational } \\
\text { normalcy (emergency, flow control, etc.), fatigue } \\
\text { (time on duty, time since break, time of day, etc.), } \\
\text { overly strong desire to complete flight } \\
\text { ("get-home-itis" } 25,26 \text { ), airspace complexity, } \\
\text { approach/departure complexity, airport } \\
\text { configuration }\end{array}$ \\
\hline $\begin{array}{l}\text { Prob. of Controlled } \\
\text { Flight Into Terrain }\end{array}$ & CFIT & Terrain, obstacles, night, (low) altitude, wind \\
\hline
\end{tabular}

Like incidents, safety metrics can be organized to facilitate communication and analysis. One possible organization, influenced by the FAA PAVE checklist mentioned earlier, groups the safety metrics into the categories of Environment-, Airspace-, and Human-centric. A different organization groups them using the ICAO/CAST taxonomy. ${ }^{24}$ We believe this latter organization facilitates a user's situational awareness by indicating what type of incident(s) may result if the risk associated with that safety metric is not sufficiently managed. The process of determining appropriate safety metrics is documented in earlier publications ${ }^{27,28}$ and hence not repeated here for conciseness. Table 1 summarizes those findings, organized into major

\footnotetext{
${ }^{\mathrm{c}}$ The Commercial Aviation Safety Team (CAST) was founded in 1998 to reduce the commercial aviation fatality risk.
} 
incident-type categories and lists the safety metrics that are consolidated into each incident-category "meta" safety metric. However the safety metrics are organized, the interface should allow the user to drill down and see any constituent safety metrics. In some use cases, the user may also want to (de-)emphasize selected constituent safety metrics. For instance, pilots of very quiet aircraft may not need alerts regarding airspace conflicts due to noise restriction areas. The interface should support customizing meta safety metric aggregation as desired.

Figure 1 shows a high-level architecture of RTSM. RTSM continuously evaluates NAS data and alerts the user if conditions defined by the safety metrics are detected or anticipated. The data includes diverse sources such as dynamic data from flight plans, surveillance information (e.g., radar tracks), weather nowcasts and forecasts, special use airspace schedules, pilot schedules, among others, as well as static data such as obstacle locations, approach/departure procedures, runway and sector layouts, etc. The safety metrics quantify conditions that expose flight(s) to harm. The safety metric computations and user notification decisions both consider context (e.g., phase of flight, type of operation, type of user, etc.) and user preferences (e.g., thresholds, hazards of interest, etc.).

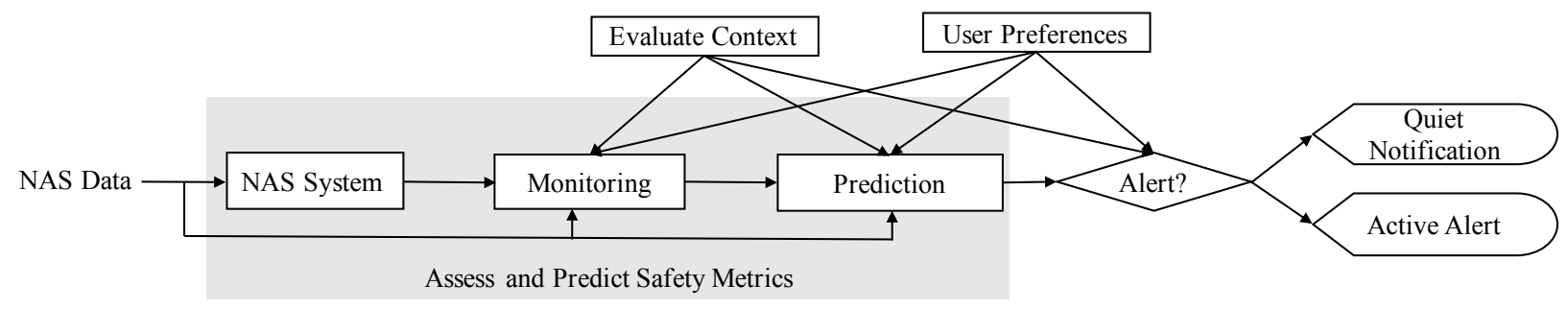

Figure 1: Real Time Safety Monitoring Architecture

\section{RTSM In Operations}

In the previous sections, we enumerated various approaches for improving aviation safety. We then introduced RTSM and discussed its expected role in reducing accidents, incidents, and close-calls by supporting operators in observing and predicting the flight context as encoded in safety metrics. In this section, we further explain the concept by describing sample use cases of RTSM. In so doing, we aim to illustrate how our work could improve real-time operations, with the ultimate goal of communicating with stakeholders to elicit the detailed requirements for RTSM.

We begin with an overview of the operators in the NAS and their responsibilities. We then present five conceptual use cases describing how RTSM could support operators' responsibilities.

\section{III.A. NAS Operators}

The NAS is a complex system, with responsibility for safe operations distributed among a number of entities. In addition to those described in this section, there are many other entities acting behind the scenes, not directly involved with real-time operations, but nevertheless, greatly influencing safety. Here, we limit the discussion only to those entities with the most proximal influence on a flight, namely the following:

- Air Traffic Control System Command Center (ATCSCC)

- Air Route Traffic Control Center (ARTCC)

- Terminal Radar Approach Control (TRACON)

- Airport Tower

- Airline: Pilot, Dispatcher, and Ramp Controller

- General Aviation: Pilot, Instructor

The Air Traffic Control System Command Center (ATCSCC) oversees daily NAS operations and determines strategies for effectively dealing with disturbances or anomalies to the system, such as widespread severe 
weather or greatly increased traffic demand in a region due to a popular sporting event (e.g., the Super Bowl). The ATCSCC is comprised of a number of interdependent positions, each with a specific responsibility, as follows:

- National Operational Manager: Oversees the implementation of the operations plan.

- Terminal Area Manager: Controls traffic flows through the NAS.

- Planner: Develops traffic management strategies.

- Severe Weather Area: Develops escape routes and pathfinder strategies during inclement weather.

- Tactical Customer Advocate: Evaluates and coordinates customer requests.

- National Operations Control Center: Monitors operational status of NAS facilities and services.

ATCSCC determines strategies applicable at the NAS level. Its plans influence the operation of flights throughout the system and over many hours.

The Air Route Traffic Control Center (ARTCC, or "Center") has more localized influence on operations in a wide area but considerably smaller than ATCSCC's area. The United States is divided into twentytwo Centers generally responsible for the enroute, typically high-altitude, phase of flights. Each Center is subdivided into a number of sectors, with one to three controllers responsible for managing traffic in each sector. Three types of Center positions of interest, that is, potential users of RTSM, and their responsibilities include the following:

- Traffic Management Unit: Balances air traffic demand with system capacity to ensure maximum efficiency and utilization of the airspace. Coordinates with ATCSCC.

- Supervisor: Assigns personnel to positions appropriate to meet traffic needs and approves special situations, such as runway or airport closures.

- Sector controller: Separates instrument flight rules (IFR) overflights, arrivals, and departures. Sequences arrivals and departures along standard instrument arrival and departure routes. Workload permitting, provides advisories to visual flight rules (VFR) traffic.

Terminal Radar Approach Control (TRACON) facilities are similar to Centers but exert even more geographically localized control. Twenty-seven TRACONs are distributed throughout the U.S. and control traffic operating nearby major commercial transport airports such as Atlanta, Chicago, Los Angeles, New York, and San Francisco. TRACON positions resemble Center positions, with sector controllers sequencing and separating traffic on instrument approach and departure procedures.

Exerting control of flights at airports are the Tower personnel. The three positions of interest to RTSM and their responsibilities are as follows:

- Supervisor: Similar responsibilities to those of Center and TRACON supervisors.

- Local controller, known as "tower" in radio communications: Issues takeoff and landing instructions ("clearances") and authorizes aircraft and other vehicle movements on or across runways.

- Ground controller: Issues taxi instructions and authorizes aircraft and other vehicle movements on the airport, except the active runway(s) or the airline ramp(s) at airports with separate ramp controllers.

RTSM is envisioned to provide airspace safety metrics for both airline and general aviation operations. There are a number of differences between these operations, with significant differences between even the various types of general aviation operations. For airline operations, we envision uses of RTSM for the following three positions:

- Pilot(s): Responsible for and final authority on the safe operation of the aircraft.

- Flight dispatchers: Share responsibility with the pilots, primarily the captain, for the safety and operational control of flights.

- Ramp controllers: Control movement of aircraft in the ramp areas, ensuring proper sequencing and separation by approving push-backs and taxi to the gate. 
For general aviation (GA), operations and incident rates vary significantly among the types of general aviation users. GA covers almost everything outside of airline and military operations. It includes corporate and charter flights with (single or dual) professional crew, business and recreational flights with a single amateur pilot, and instructional flights. Factors that affect GA safety and may contribute to the wide range in incident rates include $(i)$ differences in pilot experience and proficiency; (ii) in-flight access to additional support such as for maintenance, logistics or even a second pilot; (iii) sophistication of aircraft and avionics; and $(i v)$ frequency of recurrent training and access to high fidelity simulators for realistic emergency training. Whereas corporate and charter flights are closer to being as safe as airline operations, personal GA is significantly less safe. The enroute phase of airline flights - above much of the weather and with large margin above terrain and obstacles - is safer than terminal (i.e., departure and approach) operations. . $^{6,29,30}$ Personal GA aircraft spend a disproportionate amount of time in the the weather and near terrain and obstacles because of less capable aircraft, and near other traffic because of typical missions. With a less capable or no autopilot, the single (solo) pilot is responsible for flying the aircraft, navigating, communicating, managing systems, and anticipating the evolution of conditions through the remainder of the flight. Having RTSM automation augmenting these GA pilots could significantly improve their safety statistics.

\section{III.B. Use Cases}

We now present five sample use cases describing how RTSM could support various NAS operators. For each use case, we provide a synopsis of the operator's responsibility that is relevant to the use case, the required information to accomplish the task, a description of the use of RTSM, and additional relevant information, such as the needed prediction horizon, precision, and update rate. We also provide a graphic illustrating one way to present the information. Ultimately, users should be involved in specifying the exact requirements and designing the interface for RTSM. As for safety metrics, as mentioned previously, each user will be interested in a subset of the RTSM safety metrics. Table 2 provides a summary of the meta safety metrics of interest for each use case. Details of the safety metrics (e.g., thresholds to be used and the individual factors which should be consolidated) will differ for each user and are not specified here. Additionally, the risk aversion of each operator may differ. For example, one pilot may choose to avoid an area with a $50 \%$ probability of an adverse weather encounter, whereas another may choose to proceed toward that area but be prepared for a diversion if needed ("go take a look") for any probability less than $80 \%$. We, therefore, allow the operator to specify the notification thresholds (one or several per metric) for probability of encounter of the various hazards represented by the safety metrics.

Table 2: Safety Metrics of Interest per Use Case

\begin{tabular}{|c|c|c|c|c|c|}
\hline Safety Metric & $\begin{array}{l}\text { ATSCC } \\
\text { Planner }\end{array}$ & $\begin{array}{l}\text { TRACON } \\
\text { Approach } \\
\text { Controller }\end{array}$ & $\begin{array}{c}\text { Airport } \\
\text { "Tower" } \\
\text { Controller }\end{array}$ & $\begin{array}{c}\text { Airline } \\
\text { Pilot }\end{array}$ & $\begin{array}{c}\text { Airline } \\
\text { Dispatcher }\end{array}$ \\
\hline Prob. of Adverse Weather Encounter & $\checkmark$ & $\checkmark$ & $\checkmark$ & $\checkmark$ & $\checkmark$ \\
\hline Prob. of Airspace Conflict & $\checkmark$ & $\checkmark$ & $\checkmark$ & & $\checkmark$ \\
\hline Prob. of Malfunctioning Equipment Encounter & $\checkmark$ & $\checkmark$ & $\checkmark$ & $\checkmark$ & $\checkmark$ \\
\hline Prob. of Runway Excursion & $\checkmark$ & & & $\checkmark$ & $\checkmark$ \\
\hline Prob. of Ground Conflict & & & $\checkmark$ & & $\checkmark$ \\
\hline Prob. of Airborne Conflict & & $\checkmark$ & $\checkmark$ & $\checkmark$ & $\checkmark$ \\
\hline Prob. of Controlled Flight Into Terrain (CFIT) & & $\checkmark$ & $\checkmark$ & $\checkmark$ & $\checkmark$ \\
\hline Prob. of Excessive Pilot Workload & $\checkmark$ & $\checkmark$ & $\checkmark$ & & $\checkmark$ \\
\hline Prob. of Excessive Controller Workload & $\checkmark$ & $\checkmark$ & $\checkmark$ & & $\checkmark$ \\
\hline
\end{tabular}

\section{User 1: ATCSCC Planner}

Use Case: Develop traffic management strategies.

Required information: System-wide awareness of traffic, weather, facilities, and constraints.

Description: For each 15-min period, the Planner requests from RTSM the predicted conditions NAS-wide at the start of the flight day, allowing her to visualize and evaluate expected airspace permeability. 
Scrolling through expected traffic demand as evidenced by filed flight plans for each 15-min period, she decides whether and which flow control strategies to enact. Some of this capability already exists in automated tools used by Planners. RTSM brings it together into a single system and provides additional support with understanding of the uncertainty of the situation.

Safety Metrics: Probability of the following: Adverse Weather Encounter, Airspace Conflict, Malfunctioning Equipment Encounter, Runway Excursion, Excessive Pilot Workload, Excessive Controller Workload.

Additional Information: Some additional information includes

- Prediction horizon: 8 or more hours, enabling the operator to see if, e.g., weather will be encountered at a future operator-selected time between now and the prediction horizon, with a probability of encounter above that specified by the operator.

- Precision: 15 minutes

- Update rate: 60 minutes

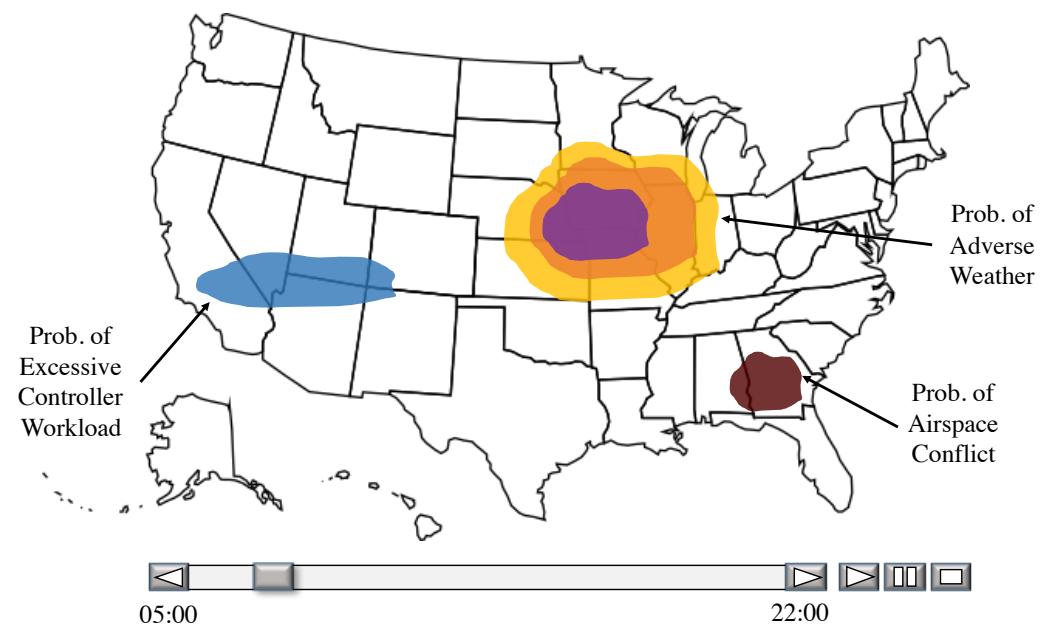

Figure 2: ATCSCC Planner Use Case

Display option: As shown in Figure 2, for the time period selected via the slider, there are three areas with predicted safety metric violations (left to right): excessive controller workload, adverse weather, and airspace conflict. The range of probabilities to display and the color-coding for each probability range are user settable. The example shows three probability range settings for adverse weather, each represented by a different color (e.g., yellow for 30\% to $40 \%$ probability of adverse weather, orange for greater than $40 \%$ but less than $75 \%$, and purple for greater than $75 \%$ ). The play, pause, and stop buttons provide the operator another method to view the evolution of conditions through a period.

\section{User 2: TRACON Approach Controller}

Use Case: Safe and efficient tactical aircraft routing.

Required Information: Sector-wide awareness of traffic, weather, facilities, and constraints.

Description: Each time new information is received, RTSM evaluates the controller-selected safety metrics (SM) for each aircraft under the controller's responsibility. If any SM exceeds its safety threshold (a function of SM value, probability, and persistence ${ }^{\mathrm{d}}$ ), an alert is provided to the controller with the type of conflict, time to conflict, and probability. Selecting an item on the alert list highlights the components involved in the conflict.

\footnotetext{
${ }^{\mathrm{d}}$ Persistence ensures that, for instance, noise in the data does not result in nuisance alerts.
} 
Safety Metrics: Probability of the following: Adverse Weather Encounter, Airspace Conflict, Malfunctioning Equipment Encounter, Airborne Conflict, Excessive Pilot Workload, Excessive Controller Workload, Controlled Flight Into Terrain.

Additional Information: Some additional information includes

- Prediction Horizon: 20 minutes

- Update rate: Event driven (e.g., weather update received, notification of equipment failure received, etc.) and surveillance update rate $(4.5 \mathrm{sec}$ or $1 \mathrm{sec})$

- Events: New aircraft, updated position or other relevant information

\begin{tabular}{|c|c|c|c|}
\hline $\begin{array}{c}\text { Time to } \\
\text { (hh:mm:ss) }\end{array}$ & Event & Aircraft & $\begin{array}{c}\text { Probability } \\
\text { (\%) }\end{array}$ \\
\hline $00: 01: 00$ & WAKE & AAL123 (DAL456) & 65 \\
\hline $00: 15: 00$ & LOS & SWA789 - UAL012 & 90 \\
\hline
\end{tabular}

(a) Example tabular format for alerts.
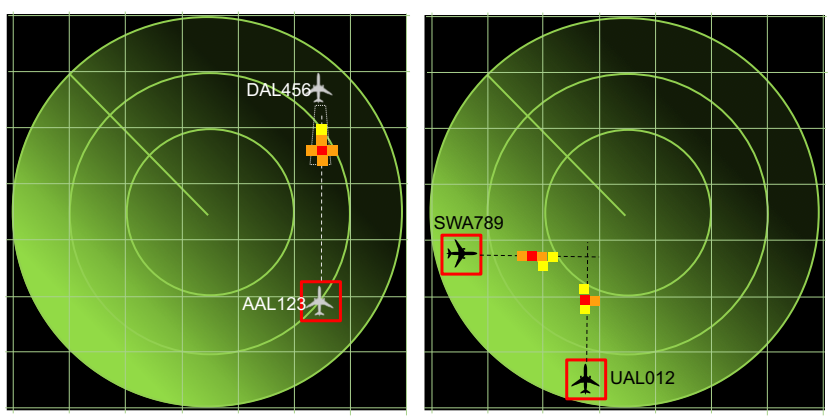

(b) Wake encounter prediction. (c) Loss of Separation prediction.

Figure 3: TRACON Approach Controller Use Case

Display option: As described above, the controller's main interface to RTSM for this use case is through alerts posted to the tabular format shown in Figure 3a. In the case shown, two airborne conflict encounters are predicted: (1) $65 \%$ probability that AAL123 will encounter wake turbulence from DAL456 in 1 minute, and (2) 90\% probability that SWA789 and UAL012 will fly closer than the lossof-separation (LOS) standard distance in 15 minutes (display is not to scale; for illustration purposes only). The controller can select a row from this table and have the conflict aircraft and areas highlighted on a traffic display, as shown in Figs. 3b and 3c. The filled squares represent the probability that the event will occur when the aircraft reaches that location, with the uncertainty, due to e.g., wind, color coded yellow, orange, and red based on user-set probability-to-color mapping thresholds.

\section{User 3: Airport Local ("Tower") Controller}

Use Case: Clear an aircraft for takeoff.

Required Information: Awareness of departure zone, arrival zone, and taxiing traffic.

Description: Taking into consideration traffic in the local area, RTSM displays the two earliest acceptable takeoff time windows for the first flight queued for departure at each runway. If an immediate takeoff violates safety metric thresholds, an alert and countdown to first available takeoff time is displayed. Prior to issuing takeoff clearance, the controller checks RTSM for an alert. If no alert is present, the controller checks the end time of the available takeoff time window to determine whether to expedite a takeoff or wait until the next opportunity.

Safety Metrics: Probability of the following: Adverse Weather Encounter, Airspace Conflict, Malfunctioning Equipment Encounter, Ground Conflict, Airborne Conflict, Excessive Pilot Workload, Excessive Controller Workload, Controlled Flight Into Terrain.

Additional Information: Some additional information includes 
- Prediction Horizon: 5 minutes

- Update rate: Event driven and surveillance update rate (4.5 sec or $1 \mathrm{sec}$ )

- Events: Aircraft ready for takeoff, location update for aircraft in the local area, weather or other relevant information updates.

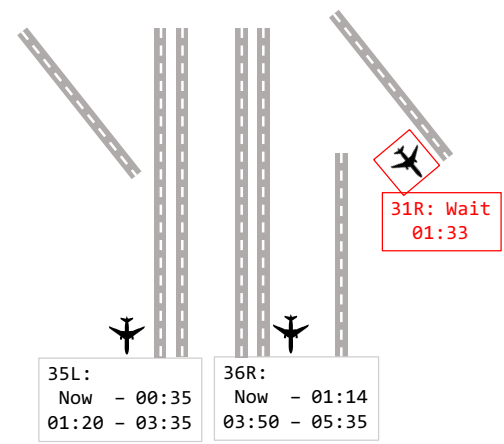

Figure 4: Tower Display Option

Display option: As can be seen in Figure 4, for this example, three aircraft are queued for departure at runways $35 \mathrm{~L}, 36 \mathrm{R}$ and $31 \mathrm{R}$. The aircraft on runways $35 \mathrm{~L}$ and $36 \mathrm{R}$ are clear of RTSM-known conflicts and can be cleared for departure within the time shown in the first window of opportunity (i.e., now to $35 \mathrm{sec}$ from now for the aircraft on 35L). The next window of opportunity is also provided in case the first window of opportunity is not utilized (for controller or pilot reasons). In contrast, RTSM is predicting a safety metric violation for the aircraft queued at runway 31R. The violation is predicted to clear in $1 \mathrm{~min} 33 \mathrm{sec}$. The controller can select the aircraft (or box) for additional information regarding the predicted violation (not shown).

\section{User 4: Airline Pilot}

Use Case: Ensure continued safety of flight on final approach.

Required Information: Awareness of safety-relevant conditions along the flight route and in the terminal area.

Description: Given the increased workload during this phase of flight, RTSM is primarily used in an intelligent alerting mode, not in an information "pull" mode. RTSM considers not just if a safety metric is violated but also the consequences of that violation in the given context. For example, if a navigational aid that is not required for approach and landing fails, RTSM only quietly posts a informative message to the crew alerting system. Alternatively, RTSM may post a caution message with an auditory beep for a prediction that the preceeding aircraft may overshoot/overrun the runway (and hence, a goaround may be necessary for "our" aircraft). Finally, an verbal caution may be issued for an airborne conflict due to a predicted wake turbulence encounter.

Safety Metrics: Probability of the following: Adverse Weather Encounter, Malfunctioning Equipment Encounter, Runway Excursion, Airborne Conflict, Controlled Flight into Terrain.

Additional Information: Some additional information includes

- Prediction Horizon: 5 minutes

- Update rate: Event driven and surveillance update rate (4.5 sec or $1 \mathrm{sec}$ )

- Events: Aircraft ready for takeoff, location update for aircraft in the local area, weather or other relevant information updates

Display Option: Two messages are shown in Figure 5. The message regarding predicted high controller workload $^{\mathrm{e}}$ is informative only and would be posted quietly in this use case. In contrast, the more critical wake turbulence caution is both posted and a verbal warning is issued.

\footnotetext{
eBecause of the numerous checks and balances that support safety, pilots and controllers need to know each other's limitations to adapt their own behavior appropriately.
} 


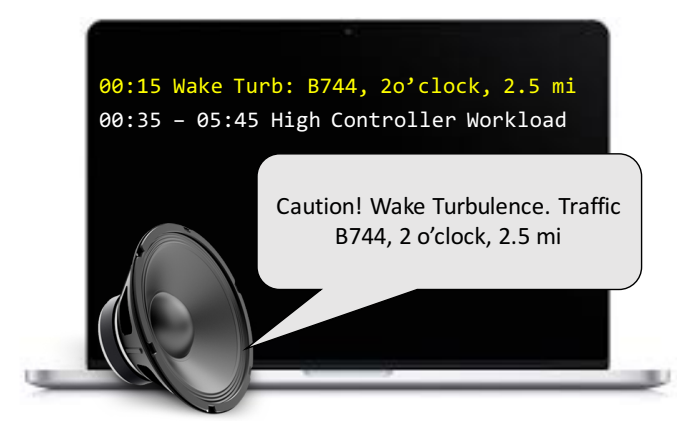

Figure 5: Pilot Display Option

\section{User 5: Aircraft Dispatcher}

Use Case: Select departure time and route efficiency for a flight near predicted convective weather. This use case demonstrates a what-if capability in which the operator is able to change the parameters used in computing safety metrics. It is also possible that the operator may want to change the assumptions of any incoming forecasts, such as when severe weather is likely to arrive.

Required Information: Awareness of safety-relevant conditions along possible routes.

Description: To evaluate potentially acceptable routes, the dispatcher can modify departure times, safety metric acceptable probability value thresholds, the safety metric aggregation function to (de-)emphasize certain metrics, and prediction for e.g., weather severity or timing. Results are shown on, for example, a heat map.

Safety Metrics: Probability of the following: Adverse Weather Encounter, Airspace Conflict, Malfunctioning Equipment Encounter, Runway Excursion, Ground Conflict, Airborne Conflict, Excessive Pilot Workload, Excessive Controller Workload, Controlled Flight Into Terrain.

Additional Information: Some additional information includes

- Prediction Horizon: Length of flight plus a user-specified safety margin, e.g., $30 \mathrm{~min}$.

- Update rate: Upon any input from the dispatcher or when new information is received.

Display Option: As shown in Figure 6, an airline dispatcher may want extensive control over specifying the parameters used in RTSM computations and in evaluating conditions over a wide area for flights of various duration and various departure times. As shown in Figure 6a, a dispatcher can specify the parameters that define individual safety metrics, such as a threshold of no closer than $20 \mathrm{mi}$ from convective weather (thunderstorms) or a criticality of "low" for determining risk from turbulence. Figure $6 \mathrm{~b}$ shows that a dispatcher may also want to limit the display of adverse conditions by specifying risk values for the meta safety metrics, for example, specifying that the combined risk of all weather conditions with a $50 \%$ or greater probability of encounter must exceed 20 (risk units to be specified). Figure $6 \mathrm{c}$ shows the selected safety metrics using the dispatcher specified colors (Figure 6b, first column) for a time selected by the slider.

\section{Conclusions}

In this paper, we presented a concept for real-time safety monitoring (RTSM) of the NAS. The problem RTSM aims to solve is providing information about current and anticipated adverse conditions, i.e., hazards to flight, in time as needed by the user to make safe yet operationally efficient decisions. A variety of customers are targeted, from the Air Traffic Control System Command Center (strategic) planners who need a broad overview of the NAS as many as 8 to 12 hours in advance, to airline dispatchers planning for and monitoring the progress of multiple flights throughout the day, to pilots and controllers responsible for ensuring safety of flights through tactical action. Various government and industry entities are providing safety guidelines and monitoring for compliance to assure safe flight operations. We benefit from their analyses, lessons learned, and best practice guidelines to select the metrics to monitor and predict. In this 


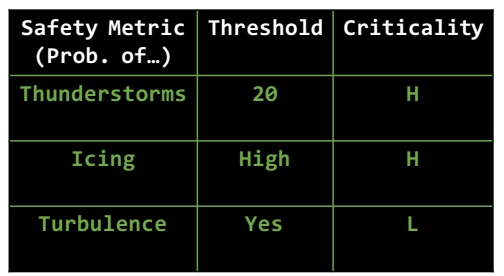

(a) Individual safety metric specification.

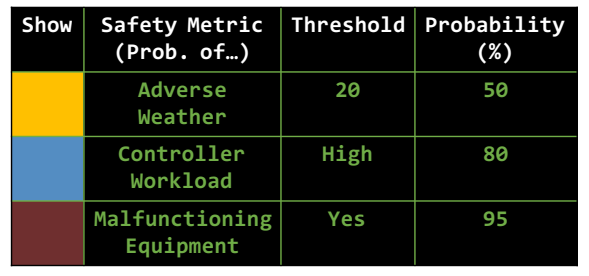

(b) Meta safety metric specification.

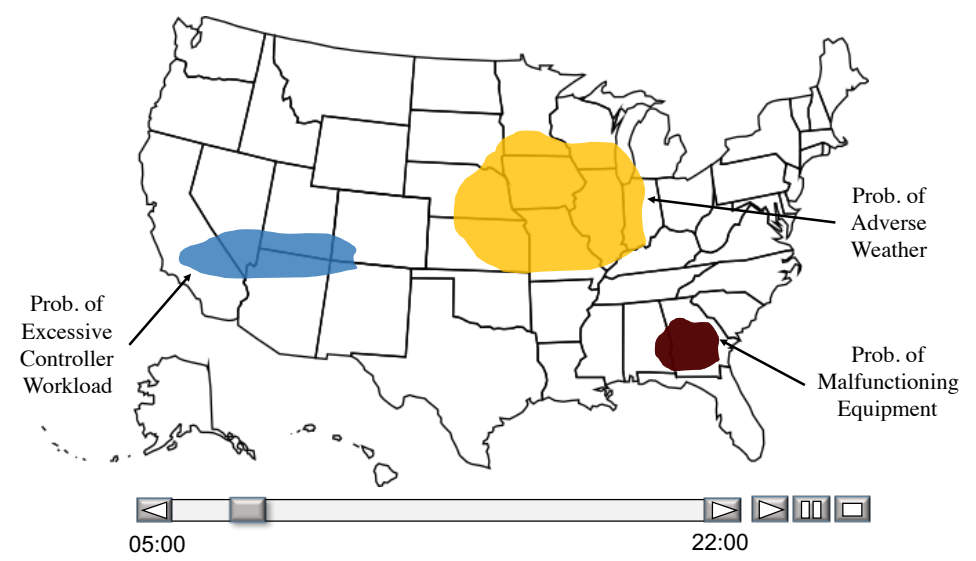

(c) Example format for visualizating the predicted situation.

Figure 6: Airline Dispatcher Use Case

paper, we presented an overview of potential metrics that are indicative of vulnerabilities in the system and target the types of conditions that may lead to incidents. We aggregate the fairly large set of metrics developed $i^{28}$ into ten meta-metrics to facilitate quick interpretation by the user, with additional details available via an envisioned drill-down capability.

The many previous efforts and related work have established a set of conditions that are indicative of higher risk operations. Unlike these efforts, we are not attempting to characterize operations and extract what conditions often lead to incidents (like NTSB, CAST, FAA, and others are doing). Nor are we attempting to compute the risk of an incident given a set of circumstances (like FAA's ISAM project aims to do). Rather, we begin with a set of conditions that these efforts have determined expose flights to vulnerabilities and may lead to incidents. We then monitor the current and anticipated state of those conditions and alert the user if some user-specified "level of acceptable safety" threshold is exceeded. Although not discussed in this paper, the information we compute can also be used to display to the user the available safety margin, which is the gap between the current value of the monitored conditions and their thresholds. ${ }^{21}$

In other related work, targeted tools have been developed to assist pilots and controllers in maintaining vigilance for adverse conditions. Examples of these tools include airborne conflict detection, airspace intrusion detection, ground proximity warning systems, and runway excursion prediction. Each of these tools warns about adverse conditions affecting one specific aircraft in a very narrow time horizon (seconds to minutes). In contrast, we provide information about conditions that increase risk to any flight transiting that airspace (including ground operations) and predict at the time horizon necessary by the user. The goal is to improve situational awareness of conditions. Moreover, we anticipate that judiciously providing ("pushing") relevant information to the user will decrease the user's workload and result in less biased information gathering.

The next step is to vet the concept with potential users. Use cases like those provided in this paper can serve to elicit the user's requirements for a monitoring system, as can discussions about what information is useful, when to present it, and how to present it to augment the user and not distract from higher priority tasks. Collaborating with the users will also lead to more detailed definitions of the safety metrics of interest to each user and the factors that should be considered and aggregated under various circumstances. 


\section{Acknowledgment}

This work was supported by the SMART-NAS project under the Airspace Operations and Safety (AOSP) program within the NASA Aeronautics Research Mission Directorate (ARMD). We would like to extend our sincere gratitude to our team for their invaluable support.

\section{References}

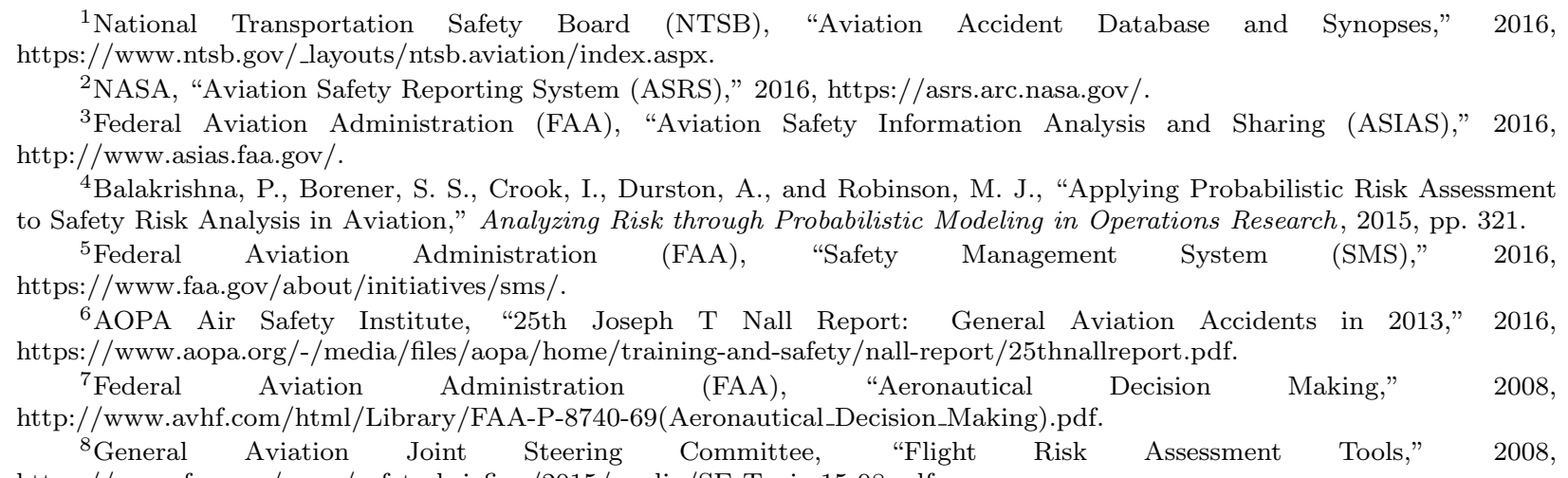
https://www.faa.gov/news/safety_briefing/2015/media/SE_Topic_15-08.pdf.

${ }^{9}$ AOPA, "Flight Risk Evaluator," 2010, https://www.aopa.org/go-fly/flight-tools.

${ }^{10}$ Smith, B., "Safety Net Inventory," Tech. Rep. To appear as NASA/TM, NASA Ames Research Center, Moffett Field, CA, USA, June 2017.

${ }^{11}$ Boeing, "Statistical Summary of Commercial Jet Airplane Accidents," 2016, www.boeing.com/news/techissues/pdf/statsum.pdf.

${ }^{12}$ Federal Aviation Administration (FAA), "FAA Aerospace Forecast, Fiscal Years 2016-2036," 2016, https://www.faa.gov/data_research/aviation/aerospace_forecasts/.

${ }^{13}$ Skybrary, "Acceptable Level of Safety," 2017, "https://www.skybrary.aero/index.php/Acceptable_Level_of_Safety".

${ }^{14}$ Glaneuski, J., "Unmanned Aircraft System (UAS) Service Demand 2015-2035 Literature Review \& Projections of Future Usage," Tech. Rep. DOT-VNTSC-DoD-13-01, U.S. Department of Transportation John A. Volpe National Transportation Systems Center, Cambridge, MA, September 2013.

${ }^{15}$ Hoekstra, J. M., van Gent, R. N., and Ruigrok, R. C., "Designing for safety: the "free flight" air traffic management concept," Reliability Engineering 85 System Safety, Vol. 75, No. 2, 2002, pp. 215-232.

${ }^{16}$ Federal Aviation Administration (FAA), "NextGEN Performance Snapshots Reference Guide," 2017, https://www.faa.gov/nextgen/snapshots/guide/.

${ }^{17}$ Boyd, J. and Col, U., "OODA loop," Destruction and Creation. Available at http://en. wikipedia. org/wiki/OODA_loop, 1995.

${ }^{18}$ Wiggins, M., "The interpretation and use of weather radar displays in aviation," Aviation Research Investigation Report. Canberra, ACT: Australian Transport Safety Bureau, 2005.

${ }^{19}$ Wiggins, M. and O'Hare, D., "Weatherwise: Evaluation of a cue-based training approach for the recognition of deteriorating weather conditions during flight," Human Factors: The Journal of the Human Factors and Ergonomics Society, Vol. 45, No. 2, 2003, pp. 337-345.

${ }^{20}$ Hunter, D. R., Martinussen, M., and Wiggins, M., "Understanding how pilots make weather-related decisions," International Journal of Aviation Psychology, Vol. 13, No. 1, 2003, pp. 73-87.

${ }^{21}$ Daigle, M., Roychoudhury, I., Spirkovska, L., Goebel, K., Sankararaman, S., Ossenfort, J., and Kulkarni, C., "RealTime Prediction of Safety Margins in the National Airspace," 17th AIAA Aviation Technology, Integration, and Operations Conference, 2017.

22 "Dictionary.com," http://www.dictionary.com/.

${ }^{23}$ ICAO, "Safety Management Manual, 3rd edition," Tech. Rep. Doc 9859 AN/474, International Civil Aviation Organization, Montreal, Quebec, Canada, 2013.

${ }^{24}$ Commercial Aviation Safety Team and International Civil Aviation Organization Common Taxonomy Team, "Aviation Occurrence Categories," 2011, http://www.icao.int/APAC/Meetings/2012_APRAST/OccurrenceCategoryDefinitions.pdf.

${ }^{25}$ Flight Safety Foundation, "Operator's Guide to Human Factors in Aviation Press-on-itis," 2017, "http://www.skybrary.aero/index.php/Press-on-itis_\%28OGHFA_BN\%29".

${ }^{26}$ Bureau d'Enquetes et d'Analyses pour la Securite de l'Aviation Civile, "The Get-home-itis syndrome," 2017, https://www.bea.aero/etudes/gethomeitis/gethomeitis.htm.

${ }^{27}$ Roychoudhury, I., Spirkovska, L., Daigle, M., Balaban, E., Sankararaman, S., Kulkarni, C., Poll, S., and Goebel, K., "Predicting Real-Time Safety of the National Airspace System," AIAA Infotech@Aerospace, 2016, p. 2131.

${ }^{28}$ Roychoudhury, I., Spirkovska, L., Daigle, M., Balaban, E., Sankararaman, S., Kulkarni, C., Poll, S., and Goebel, K., "Real-Time Monitoring and Prediction of Airspace Safety," Nov. 2015.

${ }^{29}$ ICAO, "ICAO Safety Report, 2014 Edition," Tech. rep., International Civil Aviation Organization, Montreal, Quebec, Canada, 2014. 
${ }^{30}$ AOPA Air Safety Institute, "2014-2015 GA Accident Scorecard," 2016, https://www.aopa.org//media/files/aopa/home/training-and-safety/nall-report/20142015scorecard.pdf. 